\title{
High Caloric Diet for ALS Patients: High Fat, High Carbohydrate or High Protein
}

Sarvin Sanaie ${ }^{1}$, Ata Mahmoodpoor ${ }^{2 *}$

1. Assistant professor of Nutrition. MD. PhD, Tuberculosis and Lung research center, Tabriz University of Medical Sciences, Tabriz, Iran

2. Associate Professor of Anesthesiology, Fellowship of critical Care Medicine, Cardiovascular Research Center, Tabriz University of Medical Sciences, Tabriz, Iran

ALS is a fatal motor neurodegenerative disease characterized by muscle atrophy and weakness, dysarthria, and dysphagia. The mean survival of ALS patients is three to five years, with $50 \%$ of those diagnosed dying within three years of onset (1). A multidisciplinary approach is crucial to set an appropriate plan for metabolic and nutritional support in ALS. Nutritional management incorporates a continuous assessment and implementation of dietary modifications throughout the duration of the disease. The nutritional and metabolic approaches to ALS should start when the diagnosis of ALS is made and should become an integral part of the continuous care to the patient, including nutritional surveillance, dietary counseling, management of dysphagia, and enteral nutrition when needed. Malnutrition and lean body mass loss are frequent findings in ALS patients necessitating comprehensive energy requirement assessment for these patients. Malnutrition is an independent prognostic factor for survival in ALS with a 7.7 fold increase in risk of death. Malnutrition is estimated to develop in one quarter to half of people with ALS (2). Adequate calorie and protein provision would diminish muscle loss in this vulnerable group of patients. Although appropriate amount of energy to be administered is yet to be established, high calorie diet is expected to be effective for potential improvement of survival; ALS patients do not normally receive adequate intake of energy. A growing number of clinicians suspect that a high calorie diet implemented early in their disease may help people with ALS meet their increased energy needs and extend their survival. Certain high calorie supplements appear to be safe and well tolerated by people with ALS according to studies led by Universitäts klinikum Ulm's and, appear to stabilize body weight within 3 months. In a recent study by Wills et al., intake of high-carbohydrate low-fat supplements has been recommended in ALS patients (3). They showed that patients in the highcarbohydrate/high-calorie groups gained 0.39 kg more weight per month, compared with $0.11 \mathrm{~kg}$ per month in the control group, and there was an average weight loss of $0.46 \mathrm{~kg}$ per

\section{Corresponding author:}

Ata Mahmoodpoor

Tabriz University of Medical Sciences, Tabriz, Iran

E-mail: amahmoodpoor@yahoo.com Phone: +989141160888

Receive date: 2014-04-01| Accept date: 2014-04-29 | Publish date: 2014-05-08

DOI: 10.7575/aiac.abcmed.15.03.01.02

\section{A. I}


month in the high-fat/high-calorie group. However, there are some concerns that highcarbohydrate low-fat diets might increase the risk of ALS and these findings should be interpreted with caution (4). Furthermore, according to Wills et al. high fat-high caloric diets could not be ideal regimens for these patients due to the associated gastrointestinal complications (3). Dorst and associates, in their study, showed that high caloric food supplement with high fat is suitable to establish body weight compared to high carbohydrate formula. Hence, it seems that high protein-high caloric diets could be more appropriate options for both improving negative nitrogen balance and decreasing muscle atrophy in patients with
ALS based on the pathophysiology of proteinenergy malnutrition and hypermetabolism which is thought to be due to mitochondria problem. The multifactorial pathophysiology of ALS has resulted in hypotheses that there may be subgroups of patients, eventually defined by a specific underlying etiology or clinical presentation, which selectively respond to a particular regimen. Consequently, further RCTs with larger sample size are required to clarify the best regimen for weight gain and improved survival in ALS patients and it seems that personalized nutritional support or combined regimens might be the best way and could improve the quality of life considering the complex pathophysiology of malnutrition.

\section{References}

1- Shoesmith CL, Strong MJ. Amyotrophic lateral sclerosis: Update for family physicians. Can Fam Physician. 2006; 52(12):1563-1569

2- Desport JC, Preux PM, Truong TC, et al. Nutritional status is a prognostic factor for survival in patients with ALS. Neurology. 1999; 53(5):1059-1063.

3- Wills AM, Hubbard J, Macklin EA, et al. Hypercaloric enteral nutrition in patients with amyotrophic lateral sclerosis: a randomised, double-blind, placebo-controlled phase 2 trial. Lancet. doi: 10.1016/S0140-6736(14)60222-1

4- Okamoto K, Kihira T, Kondo T, et al. Nutritional status and risk of amyotrophic lateral sclerosis in Japan. Amyotroph Lateral Scler. 2007; 8(5):300-4.

5- Dorst J, Cypionka J, Ludolph AC. High-caloric food supplements in the treatment of amyotrophic lateral sclerosis: a prospective interventional study. Amyotroph Lateral Scler Frontotemporal Degener. 2013; 14(7-8):533-6.

6- Bouteloup C, Desport JC, Clavelou P, et al. Hypermetabolism in ALS patients: an early and persistent phenomenon. J Neurol. 2009; 256(8):1236-42. 